\title{
Inhibitory Effect of Metformin Therapy on the Incidence of Colorectal Advanced Adenomas in Patients With Diabetes
}

\author{
Yo Han Kim, Ran Noh, Sun Young Cho, Seong Jun Park, Soung Min Jeon, Hyun Deok Shin, \\ Suk Bae Kim, Jeong Eun Shin \\ Department of Internal Medicine, Dankook University College of Medicine, Choenan, Korea
}

\begin{abstract}
Background/Aims: Metformin use has been associated with decreased colorectal cancer risk and mortality among diabetic patients. Recent research suggests that metformin use may decrease the incidence of colorectal adenomas in diabetic patients with previous colorectal cancer. This study aimed to assess the clinical effect of metformin use on the development of colorectal adenomas in diabetic patients without previous colorectal cancer. Methods: Among 604 consecutive diabetic patients who underwent colonoscopic surveillance after initial colonoscopy between January 2002 and June 2012, 240 patients without previous colorectal cancer were enrolled in this study and were divided in two groups: 151 patients receiving metformin and 89 patients not receiving metformin. Patient demographics and clinical characteristics as well as the colorectal adenoma incidence rate were retrospectively analyzed. Results: The incidence rate of total colorectal adenomas was not different according to metformin use $(P=0.349)$. However, the advanced adenoma incidence rate was significantly lower in the metformin group compared with the non-metformin group (relative risk [RR], $0.09 ; P=0.011$ ). Metformin use was independently associated with a decreased incidence of advanced colorectal adenomas after adjustment for clinically relevant factors $(\mathrm{RR}, 0.072 ; P=0.016)$. In addition, the cumulative development rate of advanced adenomas during follow-up was significantly lower in the metformin group compared with the non-metformin group $(P=0.007)$. Conclusions: Metformin use in diabetic patients without previous colorectal cancer is associated with a lower risk of advanced colorectal adenomas. (Intest Res 2015;13:145-152)
\end{abstract}

Key Words: Advanced colorectal adenoma; Diabetes mellitus; Incidence; Metformin

\section{INTRODUCTION}

Colorectal cancer is the third most common cancer worldwide, and has been showing an increasing incidence in Korea. ${ }^{1,2}$ As most colorectal cancers arise from adenomas, colorectal adenomas are highly prone to develop into colorectal carcinomas. $^{3,4}$

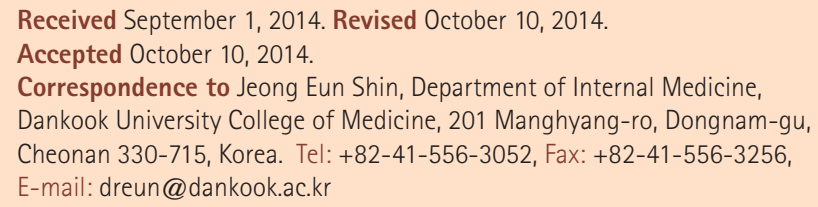

Financial support: None. Conflict of interest: None.
Diabetes mellitus (DM) has been recognized as a risk factor for all cancer incidence and mortality rates, and has been significantly associated with the prevalence of breast and colon cancers. ${ }^{5-7}$ It has been proposed that DM may also have adverse effects on the prognosis of colon cancer, that hypoglycemic agents such as insulin, sulfonylureas and others are effective in decreasing colon cancer mortality and incidence, and that hyperinsulinemia is involved in the development of

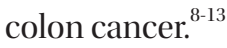

Metformin regulates blood sugar primarily by inhibiting hepatic gluconeogenesis and by reducing insulin resistance in peripheral tissues. Metformin activates AMP-activated protein kinase (AMPK) in the liver, and AMPK activation has an inhibitory effect on cancer cell growth and new blood

\footnotetext{
๑ Copyright 2015. Korean Association for the Study of Intestinal Diseases. All rights reserved.

This is an Open Access article distributed under the terms of the Creative Commons Attribution Non-Commercial License (http://creativecommons.org/licenses/by-nc/3.0)

which permits unrestricted non-commercial use, distribution, and reproduction in any medium, provided the original work is properly cited.
} 
vessel formation by prohibiting activation of mammalian target of rapamycin (mTOR). ${ }^{14}$ The results of an animal study suggested that metformin use lowered the risk of colorectal adenomas, pre-cancerous lesions, in addition to lowering colorectal cancer risk. ${ }^{15,16}$ A recent study reported that the incidence of colorectal adenomas was reduced significantly in diabetic patients receiving metformin compared with those not receiving metformin among patients under follow-up after a diagnosis of colorectal cancer. This outcome suggests that metformin has the potential to lower the incidence of colorectal adenomas. ${ }^{17}$

The current study was performed to identify the effect of metformin use on colorectal adenoma incidence in diabetic patients without previous colorectal cancer.

\section{METHODS}

\section{Materials}

We initially identified as possible study subjects 604 patients who underwent more than one surveillance colonoscopy from among 2,446 diabetic patients who underwent screening colonoscopy in the Dankook University Hospital between January 2002 and June 2012. We excluded patients who did not undergo surveillance colonoscopy, those who had an incomplete colonoscopy due to poor bowel cleanliness, and those who received a repeat colonoscopy within a year due to a previous incomplete colonoscopy. We finally included 240 patients, after excluding 40 patients who underwent colonoscopic surveillance to follow up gastrointestinal bleeding or IBD, 4 patients who had an incomplete colonoscopy, 216 patients for whom there was no available information on diabetes medications in the medical records, and 104 patients who had been previously diagnosed with colorectal cancer or had a history of colon resection. Of these 240 study subjects, 151 patients received metformin and 89 patients did not. With a retrospective review of the medical records, the demographics and clinical characteristics of the patients were analyzed, including age, sex, BMI, family history of colorectal cancer, DM duration, follow-up duration, smoking and drinking history, serum cholesterol, and glycated hemoglobin (HbAlc). In addition to history of metformin use, patients' previous history of anti-diabetic drugs (insulin, thiazolidinediones) and aspirin use were also examined.

\section{Methods}

All examinations were conducted by 6 experienced endoscopists using a standard colonoscope (CF-Q240I and CF-Q260AI; Olympus, Tokyo, Japan). All adenomas detected during screening colonoscopy were removed in all patients. Even though the interval between follow-up colonoscopies varied, surveillance colonoscopy was performed at least once. The time of occurrence, number and size of adenomas, and biopsy outcomes were examined. Adenoma size was measured using biopsy forceps during colonoscopy. Advanced adenomas were defined as an adenoma larger than $10 \mathrm{~mm}$ or an adenoma of any size with a villous or tubulovillous component, or high-grade dysplasia. Hyperplastic or inflammatory polyps were discriminated by the absence of adenomatous polyps. The BMI of patients was classified into normal $\left(\leq 25 \mathrm{~kg} / \mathrm{m}^{2}\right)$, overweight $\left(25-30 \mathrm{~kg} / \mathrm{m}^{2}\right)$, and obese $\left(\geq 30 \mathrm{~kg} / \mathrm{m}^{2}\right)$. Based on medical records, patients who used metformin for more than 6 months at minimum were categorized into the metformin group. This study was performed after gaining approval from the Institutional Review Board (IRB) of the Dankook University Hospital.

\section{Statistical Analysis}

This study analyzed the incidence of colorectal adenomas according to metformin use, and assessed the inhibitory effect of metformin on advanced adenomas. All analyses of statistical data were performed using SPSS version 18.0 for Windows (SPSS Inc., Chicago, IL, USA). For univariate analysis, both Student's $t$-test and Pearson's $\chi^{2}$ test were used, and for multivariate analysis, a Cox proportional hazards model was used. In addition, a Kaplan-Meier model was used to compare cumulative development rates of adenomas. $P$-values of less than 0.05 were considered statistically significant.

\section{RESULTS}

\section{Demographics and Clinical Characteristics of Subjects}

The mean age was $61.6 \pm 10.3$ years in the non-metformin group and $58.8 \pm 9.9$ years in the metformin group. Although the mean age was higher in the non-metformin group, no difference was found between the two groups using 60 years of age as the cut-off value. There were no differences between the two groups in sex, HbAlc, serum cholesterol, BMI, DM duration, or family history of colorectal cancer. Aspirin was more frequently used in the metformin group, while insulin 
was more commonly used in the non-metformin group. However, there was no difference in thiazolidinedione use between the two groups. No difference was found in smoking status between the two groups, while the rate of alcohol consumption was significantly higher in the metformin group. Screening colonoscopy revealed no difference in the average number of colorectal adenomas and the number of adenomas between the two groups (Table 1).

\section{Metformin Use, Adenoma Incidence Rate, and Risk Factors}

The mean duration of metformin use was $3.8 \pm 2.4$ years. The mean length of follow-up was $51.9 \pm 27.6$ months in

Table 1. Demographic and Clinical Characteristics of Patients According to Metformin Use

\begin{tabular}{|c|c|c|c|}
\hline Variables & Metformin group $(n=151)$ & Non-metformin group $(n=89)$ & $P$-value \\
\hline Age (yr) & $58.8 \pm 9.9$ & $61.6 \pm 10.3$ & 0.036 \\
\hline Male gender & $105(69.5)$ & $60(67.4)$ & 0.420 \\
\hline $\mathrm{HbA1c}$ & $7.7 \pm 1.7$ & $7.7 \pm 1.7$ & 0.989 \\
\hline Cholesterol (mg/dL) & $168.7 \pm 37.5$ & $178.7 \pm 44.3$ & 0.064 \\
\hline DM duration (yr) & $8.2 \pm 7.6$ & $10.0 \pm 8.5$ & 0.091 \\
\hline Family history of CRC & $5(3.3)$ & $2(2.2)$ & 0.608 \\
\hline Aspirin use & $113(74.8)$ & $52(58.4)$ & 0.006 \\
\hline Insulin use & $17(11.3)$ & $27(30.3)$ & 0.000 \\
\hline Thiazolidinedione use & $5(3.3)$ & $2(2.2)$ & 0.466 \\
\hline $\mathrm{BMI}\left(\mathrm{kg} / \mathrm{m}^{2}\right)$ & $25.2 \pm 3.0$ & $25.2 \pm 3.3$ & 0.365 \\
\hline Normal $(\leq 25)$ & $72(47.7)$ & $45(50.6)$ & \\
\hline Overweight (25-30) & $70(46.3)$ & 35 (39.3) & \\
\hline Obese $(\geq 30)$ & $9(6.0)$ & $9(10.1)$ & \\
\hline Smoking & & & 0.865 \\
\hline None & $95(62.9)$ & $53(59.6)$ & \\
\hline Ex-smoker & $24(15.9)$ & $16(18.0)$ & \\
\hline Current smoker & $32(21.2)$ & $20(22.5)$ & \\
\hline Alcohol use & $90(59.6)$ & 35 (39.3) & 0.003 \\
\hline Mean number of baseline colorectal adenomas & $1.46 \pm 2.21$ & $1.46 \pm 2.02$ & 0.990 \\
\hline Number of baseline adenomas & $86(57.0)$ & $48(53.9)$ & 0.374 \\
\hline Non-advanced adenomas & $78(51.7)$ & $42(47.2)$ & 0.462 \\
\hline Advanced adenomas & $8(5.3)$ & $6(6.7)$ & 0.473 \\
\hline
\end{tabular}

Values are presented as mean \pm SD or $n(\%)$.

$\mathrm{HbA1c}$, glycated hemoglobin; DM, diabetes mellitus; CRC, colorectal cancer.

Table 2. Comparison of Follow-Up Duration and Total Number of Follow-Up Colonoscopies Between the Metformin and Non-Metformin Groups

\begin{tabular}{cccc}
\hline \multicolumn{1}{c}{ Variables } & Metformin group (n=151) & Non-metformin group (n=89) & $P$-value \\
\hline Follow-up duration (mo) & $51.9 \pm 27.6$ & $53.4 \pm 26.0$ & 0.671 \\
No. of follow-up colonoscopies & & & 0.352 \\
1 & $93(61.6)$ & $53(59.6)$ \\
2 & $39(25.8)$ & $19(21.3)$ \\
$\geq 3$ & $19(12.6)$ & $17(19.1)$ \\
\hline
\end{tabular}

Values are presented as mean \pm SD or $n(\%)$. 
Table 3. Comparison of Adenoma Incidence Rate Between the Metformin and Non-Metformin Groups

\begin{tabular}{lccccc}
\hline \multicolumn{1}{c}{ Variables } & Metformin group $(\mathrm{n}=\mathbf{1 5 1 )}$ & Non-metformin group $(\mathrm{n}=\mathbf{8 9})$ & $\mathrm{RR}$ & $\mathbf{9 5 \%} \mathrm{Cl}$ & $\boldsymbol{P}$-value \\
\hline Total adenomas & $80(53.0)$ & $53(59.6)$ & 0.77 & $0.450-1.301$ & 0.349 \\
Non-advanced adenomas & $79(52.3)$ & $47(52.8)$ & 0.98 & $0.580-1.657$ & 0.941 \\
Advanced adenomas & $1(0.7)$ & $6(6.7)$ & 0.09 & $0.011-0.779$ & 0.011 \\
\hline
\end{tabular}

Values are presented as $n(\%)$.

Table 4. Multivariate Analysis of Total Adenoma and Advanced Adenoma Incidence Rate

\begin{tabular}{|c|c|c|c|c|c|c|}
\hline \multirow{2}{*}{ Variables } & \multicolumn{2}{|c|}{ Total adenomas } & \multirow{2}{*}{$P$-value } & \multicolumn{2}{|c|}{ Advanced adenomas } & \multirow{2}{*}{$P$-value } \\
\hline & RR & $95 \% \mathrm{Cl}$ & & RR & $95 \% \mathrm{Cl}$ & \\
\hline Metformin use (yes vs. no) & 0.866 & $0.453-1.623$ & 0.654 & 0.072 & $0.008-0.610$ & 0.016 \\
\hline Sex (male vs. female) & 1.967 & $1.102-3.513$ & 0.022 & 2.513 & $0.194-32.528$ & 0.481 \\
\hline Age (<60 vs. $\geq 60)$ & 1.273 & $0.696-2.331$ & 0.433 & 4.558 & $0.452-45.942$ & 0.198 \\
\hline $\mathrm{BMI}(\leq 25 \mathrm{~kg} / \mathrm{m} 2 \mathrm{vs} .>25 \mathrm{~kg} / \mathrm{m} 2)$ & 1.051 & $0.516-2.145$ & 0.452 & 0.207 & $0.025-1.722$ & 0.145 \\
\hline Aspirin use (yes vs. no) & 1.704 & $0.770-3.772$ & 0.188 & 1.331 & $0.195-9.084$ & 0.771 \\
\hline Smoking (none vs. ex/current smoker) & 1.051 & $0.508-1.590$ & 0.715 & 2.783 & $0.340-22.771$ & 0.340 \\
\hline Alcohol use (no vs. yes) & 1.027 & $0.492-2.145$ & 0.943 & 0.975 & $0.119-7.985$ & 0.987 \\
\hline
\end{tabular}

Statistical analysis was performed by using the Cox proportional hazards model.

the metformin group and $53.4 \pm 26.0$ months in the nonmetformin group. There was no difference between the two groups in the number of follow-up colonoscopies, with the patients divided according to whether they underwent the procedure one, two, or more than three times (Table 2). No difference was found in the total incidence rate of adenomas between the two groups. The incidence rate of advanced adenomas was $0.7 \%$ ( 1 out of 151 patients) in the metformin group and $6.7 \%$ (6/89 patients) in the non-metformin group, demonstrating a lower incidence in the metformin group (RR, 0.09; 95\% CI, 0.011-0.779; $P=0.011$ ) (Table 3). Advanced adenomas detected in the metformin group were greater than $1 \mathrm{~cm}$ in size. Meanwhile, advanced adenomas detected in the non-metformin group either were greater than $1 \mathrm{~cm}$, in 4 cases, or featured villous histology or focal high-grade dysplasia, in one case each. In a multivariate analysis using a Cox proportional hazards model, no difference was found in the risk of adenoma development according to metformin use. The risk of colorectal adenoma was increased in men (RR, 1.967; 95\% CI, 1.102-3.513; $P=0.022$ ). Metformin use was an independent factor associated with a decreased incidence of advanced colorectal adenomas in diabetic patients (RR, 0.072; 95\% CI, 0.008-0.610; $P=0.016$ ) (Table 4).

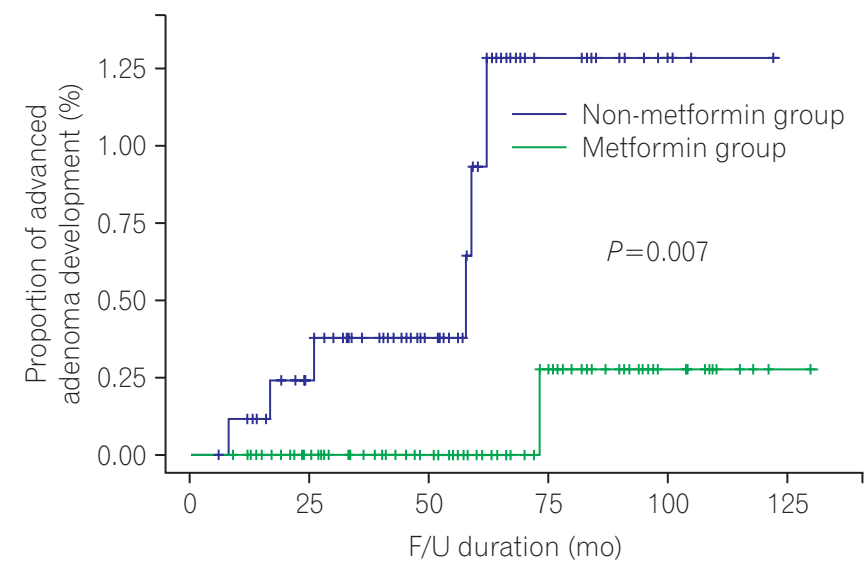

Fig. 1. Cumulative development rates of advanced adenomas according to metformin use. The rates were significantly lower in the metformin group compared with the non-metformin group during follow-up (F/U) $(P=0.007)$.

\section{Cumulative Development Incidence Rates of Total and Advanced Adenomas According to Metformin Use}

No difference was found in the cumulative development rates of total adenomas between the metformin and nonmetformin groups. The cumulative development rates of advanced adenomas were significantly lower in the metformin group compared with the non-metformin group $(P=0.007)$ (Fig. 1). 


\section{DISCUSSION}

In this study, the use of metformin was found to lower the risk of advanced colorectal adenomas in diabetic patients without previous colorectal cancer. Moreover, metformin use is associated with a decrease in the cumulative development rate of advanced adenomas.

Previous studies have suggested that the use of metformin helps to increase the survival rate in patients with colorectal cancer, as well as lowering the risk of colorectal cancer development. ${ }^{18-20}$ A number of recent studies have also reported that metformin is effective in reducing the risk of colorectal adenomas. In a previous study on patients who underwent screening colonoscopy, 148 out of 405 diabetic patients were receiving metformin, and the incidence of colorectal adenoma was lower in the metformin group $(\mathrm{OR}$, $0.55 ; 95 \% \mathrm{CI}, 0.34-0.87 ; P=0.011) .{ }^{21}$ However, the outcome of this study might be limited, because the use of other drugs that might have affected the incidence of adenomas, such as aspirin, among others, was not reported and the groups were not equalized. In a larger-scale study examining 3,105 patients, 912 diabetic patients received metformin, and the risk of adenomas was reduced in the metformin group (OR, 0.74 ; 95\% CI, 0.55-0.98; $P=0.03){ }^{22}$ However, the metformin group in the study was defined as patients receiving metformin for only a minimum of 1 month before the time of colonoscopy and the medication period was relatively short, an average of $14.7 \pm 18.2$ months, and unequal. In addition, in a recent study on 240 diabetic patients who underwent surgery due to colorectal cancer, metformin use reduced the incidence of colorectal adenomas (OR, 0.27; 95\% CI, 0.100-0.758; $P=0.012) .{ }^{17}$ However, the inhibitory effect of metformin required further verification, because the study comprised colorectal cancer patients with a high risk of colorectal adenomas.

Even though the present study also involved diabetic patients receiving metformin, patient groups were equalized through the removal of their adenomas by means of screening colonoscopy to allow the assessment of the incidence of colonic adenomas according to metformin use, unlike previous studies. Moreover, this study excluded patients diagnosed with colorectal cancer who were at a high risk of developing colorectal adenomas, and examined patients' drug histories including insulin and aspirin use, which could affect adenoma incidence.

Metformin is a widely used anti-diabetic drug and, unlike selective cyclooxygenase-2 inhibitors and other drugs, it has quite a few side effects. For this reason, metformin is an appropriate chemopreventive agent for colorectal cancer. Although the mechanism of metformin that reduces cancer incidence remains unclear, various mechanisms have been proposed. Metformin's mechanisms of action primarily prohibit the cell cycle, cellular growth, and angiogenesis by inhibiting the mTOR pathway. ${ }^{14}$ Other metformin-mediated effects include reduction in insulin-like growth factor, ${ }^{23}$ hindrance of human epidermal growth factor receptor 2 signaling, ${ }^{24}$ and cell cycle arrest, ${ }^{25}$ among others. Some studies have suggested that metformin has inhibitory effects on tumor growth through the activation of $\mathrm{p} 53 .^{26}$

The exact mechanism responsible for the reduced incidence and cumulative development rates of advanced adenomas in the current study has not yet been clarified. It is anticipated that the results are congruent with the findings of previous studies ${ }^{27}$ that metformin reduces the number of aberrant crypt foci (ACF) and dysplastic ACF and suppresses colonic epithelial proliferation. Furthermore, activated p53, which plays an important role in the advanced adenoma stage, seems to be associated. However, metformin had no influence on total adenoma incidence in this study. This outcome contradicts that of a previous study on patients with colorectal cancer. ${ }^{17}$ The result is attributed to the fact that it remains unclear at which stage and by what extent metformin influences tumor growth, because metformin may affect the progression of normal tissues to adenoma and cancer throughout all stages, from early to advanced stages. Other possibilities are that the exact dose of metformin actually used might not have been fully reflected in a retrospective study performed based on medical records, and a difference might have been present in the detection rate of adenomas by various endoscopists, although 6 experienced endoscopists performed all procedures. Some studies with relatively small sample sizes have reported that there is no association of metformin use with colorectal adenoma incidence. ${ }^{28}$

The exact amount and duration of metformin use for cancer prevention have not yet been clarified. The medication period of metformin varies in previous studies on the effect of metformin on colorectal adenoma and cancer incidence. Lee et al. ${ }^{19}$ defined the metformin group as patients with continued use of metformin before and after the diagnosis of colorectal cancer. Garrett et al. ${ }^{18}$ compared and analyzed two groups classified according to metformin use at the time of colorectal cancer detection. In both studies, the duration of metformin use varied and was unclear. In a comparative study on colorectal adenoma development and metformin use, there were no standards for the minimum medication period of metformin and the mean duration 
was 3.91 years. ${ }^{21}$ Based on the results of a study ${ }^{27}$ that found that the number of ACF was significantly reduced 1 month after metformin use, Cho et al. ${ }^{22}$ have recently defined the metformin group as patients who have used metformin for more than a month. The mean duration of metformin was $14.7 \pm 18.2$ months. No studies have reported that several possible mechanisms for metformin-mediated effects on tumor growth vary in degree according to the duration of metformin use, and the standard dose of metformin sufficient to induce its inhibitory effect on cancer development remains unclear. Moreover, the longer the duration of metformin use, the more likely it is that the medication will suppress the development of colorectal adenomas and cancer. However, no studies have yet investigated the question of a quantitative relationship between the duration of metformin use and tumor suppression. In the present study, the metformin group was defined as patients for whom the medical records showed use of metformin for more than 6 months at a minimum on a regular basis. Although a previous study classified patients with metformin use for a minimum of 1 month as the metformin group, the authors of this study believed that regular use of metformin for 6 months at a minimum is necessary to activate the mechanism of metformin to inhibit colorectal adenomas. In addition, the mean duration of metformin use in the current study was 3.8 years, similar to the longest mean duration in earlier studies ( 3.9 years). ${ }^{21}$

In a previous study on non-diabetic patients, the average number of ACF was considerably reduced $(8.78 \pm 6.45$ vs. $5.11 \pm 4.99, P=0.007)$ and colonic epithelial proliferation was suppressed in the metformin group at a dose of $250 \mathrm{mg}$ daily for a month compared with the control group. As no changes were observed in the non-metformin group, a low dose of metformin is suggested to be effective in preventing colorectal cancer. ${ }^{27}$ Further studies are warranted to clarify the appropriate amount, method, and duration of metformin use to maximize its inhibitory effect on colorectal adenomas or cancer and minimize adverse events. Currently, a double-blind, randomized study on non-diabetic patients with recent polypectomy is in progress in order to compare the effects of metformin on colorectal adenomas between a metformin group at a dose of $250 \mathrm{mg}$ daily and a placebo group for a year. $^{29}$

Even though aspirin is known to be effective in reducing colorectal adenoma incidence, ${ }^{30}$ it had no influence in the current study. In addition, although insulin and thiazolidinediones have been found to affect adenoma incidence, ${ }^{31,32}$ they showed no effect in this study. However, it is possible that the effects of these anti-diabetic drugs on colorectal ad- enoma incidence have not been identified, because the dose and duration of these drugs were not accurately examined in this study with a relatively small sample size.

There are several limitations of the present study.

First, randomized allocation was not used to prove the effect of metformin, and the number of surveillance colonoscopies varied from 1 to 3 or more. As there were no differences in the total follow-up duration and the numbers of surveillance colonoscopies between the two groups, those problems could have been partially improved.

Second, errors may be present in data collection in a retrospective study performed based on medical records. Moreover, this study did not examine patients' medical-history in relation to other drugs that could influence the incidence of colonic adenomas, such as aspirin. In particular, we included subjects without knowing their medication period and total dose of metformin before inclusion in this study. Thus, the effect of metformin exposure prior to this research cannot be ignored.

Third, patients with medical records confirming 6 months of regular use of metformin at a minimum were defined as the metformin group. The mean duration was 3.8 years. However, there was an unequal distribution of duration of metformin use, and it is also possible that this period of time was insufficient to verify its inhibitory effect.

Fourth, the quality of colonoscopy was not fully reflected. The degree of quality control including colonoscopy withdrawal time and adenoma detection rate was not sufficiently considered. Although 6 experienced endoscopists performed all procedures throughout the investigation, it is possible that the adenoma detection rate could have been influenced by different endoscopists.

Finally, this research was a single-center study involving a small sample size. The patient group had relatively wellcontrolled diabetes with a median HbAlc value of $7.7 \%$, and the average periods of DM duration were not long at 8.2 and 10 years for the metformin and non-metformin groups, respectively. For these reasons, the small sample group was insufficient to represent an entire diabetic patient group. Taking into consideration the fact that DM and insulin resistance have significant impacts on adenoma incidence, the incidence rate of adenomas was lower in the current study because a large number of patients with a relatively mild diabetic condition were included. Hence, it is possible that metformin had no inhibitory effect on the total adenoma in cidence rate. Therefore, this study was limited in its ability to clearly prove the effect of metformin as a chemopreventive agent for colorectal adenoma incidence in diabetic patients. 
To sum up the above study findings, although the authors were unable to clarify the mechanisms of metformin and included diabetic patients only, this study was meaningful in that, unlike other previous studies, it verified the potential of metformin as a preventive agent for patients with an average risk of developing colorectal cancer. We suggest that prospective, randomized, large-scale studies are warranted to further explore the chemopreventive efficacy of metformin on colorectal cancer.

\section{REFERENCES}

1. Siegel R, Naishadham D, Jemal A. Cancer statistics, 2012. CA Cancer J Clin 2012;62:10-29.

2. Jung KW, Park S, Kong HJ, et al. Cancer statistics in Korea: incidence, mortality and survival in 2006-2007. J Korean Med Sci 2010;25:1113-1121.

3. Vogelstein B, Fearon ER, Hamilton SR, et al. Genetic alterations during colorectal-tumor development. N Engl J Med 1988;319:525-532.

4. Maisonneuve P, Botteri E, Lowenfels AB. Five-year risk of colorectal neoplasia after negative colonoscopy. N Engl J Med 2008;359:2611-2612.

5. Levine W, Dyer AR, Shekelle RB, Schoenberger JA, Stamler J. Post-load plasma glucose and cancer mortality in middle-aged men and women. 12-year follow-up findings of the Chicago Heart Association Detection Project in Industry. Am J Epidemiol 1990;131:254-262.

6. Saydah SH, Loria CM, Eberhardt MS, Brancati FL. Abnormal glucose tolerance and the risk of cancer death in the United States. Am J Epidemiol 2003;157:1092-1100.

7. Weiderpass E, Gridley G, Nyren O, Ekbom A, Persson I, Adami HO. Diabetes mellitus and risk of large bowel cancer. J Natl Cancer Inst 1997;89:660-661.

8. Coughlin SS, Calle EE, Teras LR, Petrelli J, Thun MJ. Diabetes mellitus as a predictor of cancer mortality in a large cohort of US adults. Am J Epidemiol 2004;159:1160-1167.

9. Meyerhardt JA, Catalano PJ, Haller DG, et al. Impact of diabetes mellitus on outcomes in patients with colon cancer. J Clin Oncol 2003;21:433-440.

10. Currie CJ, Poole CD, Gale EA. The influence of glucose-lowering therapies on cancer risk in type 2 diabetes. Diabetologia 2009;52:1766-1777.

11. Dehal AN, Newton CC, Jacobs EJ, Patel AV, Gapstur SM, Campbell PT. Impact of diabetes mellitus and insulin use on survival after colorectal cancer diagnosis: the Cancer Prevention StudyII Nutrition Cohort. J Clin Oncol 2012;30:53-59.
12. Seow A, Yuan JM, Koh WP, Lee HP, Yu MC. Diabetes mellitus and risk of colorectal cancer in the Singapore Chinese Health Study. J Natl Cancer Inst 2006;98:135-138.

13. Atchison EA, Gridley G, Carreon JD, Leitzmann MF, McGlynn KA. Risk of cancer in a large cohort of U.S. veterans with diabetes. Int J Cancer 2011;128:635-643.

14. Sarbassov DD, Ali SM, Sabatini DM. Growing roles for the mTOR pathway. Curr Opin Cell Biol 2005;17:596-603.

15. Hosono K, Endo H, Takahashi H, et al. Metformin suppresses azoxymethane-induced colorectal aberrant crypt foci by activating AMP-activated protein kinase. Mol Carcinog 2010; 49:662-671.

16. Tomimoto A, Endo H, Sugiyama M, et al. Metformin suppresses intestinal polyp growth in $A p c^{\mathrm{Min} /+}$ mice. Cancer Sci 2008; 99:2136-2141.

17. Lee JH, Jeon SM, Hong SP, Cheon JH, Kim TI, Kim WH. Metformin use is associated with a decreased incidence of colorectal adenomas in diabetic patients with previous colorectal cancer. Dig Liver Dis 2012;44:1042-1047.

18. Garrett CR, Hassabo HM, Bhadkamkar NA, et al. Survival advantage observed with the use of metformin in patients with type II diabetes and colorectal cancer. Br J Cancer 2012; 106:1374-1378.

19. Lee JH, Kim TI, Jeon SM, Hong SP, Cheon JH, Kim WH. The effects of metformin on the survival of colorectal cancer patients with diabetes mellitus. Int J Cancer 2012;131:752-759.

20. Zhang ZJ, Zheng ZJ, Kan H, et al. Reduced risk of colorectal cancer with metformin therapy in patients with type 2 diabetes: a meta-analysis. Diabetes Care 2011;34:2323-2328.

21. Kanadiya MK, Gohel TD, Sanaka MR, Thota PN, Shubrook JH, Jr. Relationship between type-2 diabetes and use of metformin with risk of colorectal adenoma in an American population receiving colonoscopy. J Diabetes Complications 2013;27:463466.

22. Cho YH, Ko BM, Kim SH, et al. Does metformin affect the incidence of colonic polyps and adenomas in patients with type 2 diabetes mellitus? Intest Res 2014;12:139-145.

23. Pollak M. Insulin and insulin-like growth factor signalling in neoplasia. Nat Rev Cancer 2008;8:915-928.

24. Vazquez-Martin A, Oliveras-Ferraros C, Menendez JA. The antidiabetic drug metformin suppresses HER2 (erbB-2) oncoprotein overexpression via inhibition of the mTOR effector p70S6K1 in human breast carcinoma cells. Cell Cycle 2009; 8:88-96.

25. Ben Sahra I, Laurent K, Loubat A, et al. The antidiabetic drug metformin exerts an antitumoral effect in vitro and in vivo through a decrease of cyclin D1 level. Oncogene 2008;27:35763586. 
26. Buzzai M, Jones RG, Amaravadi RK, et al. Systemic treatment with the antidiabetic drug metformin selectively impairs p53deficient tumor cell growth. Cancer Res 2007;67:6745-6752.

27. Hosono K, Endo H, Takahashi H, et al. Metformin suppresses colorectal aberrant crypt foci in a short-term clinical trial. Cancer Prev Res (Phila) 2010;3:1077-1083.

28. Chung YW, Han DS, Park KH, Eun CS, Yoo KS, Park CK. Insulin therapy and colorectal adenoma risk among patients with Type 2 diabetes mellitus: a case-control study in Korea. Dis Colon Rectum 2008;51:593-597.

29. Higurashi T, Takahashi H, Endo H, et al. Metformin efficacy and safety for colorectal polyps: a double-blind randomized controlled trial. BMC Cancer 2012;12:118.
30. Baron JA, Cole BF, Sandler RS, et al. A randomized trial of aspirin to prevent colorectal adenomas. N Engl J Med 2003;348:891899.

31. Campbell PT, Deka A, Jacobs EJ, et al. Prospective study reveals associations between colorectal cancer and type 2 diabetes mellitus or insulin use in men. Gastroenterology 2010;139:11381146.

32. Okumura T. Mechanisms by which thiazolidinediones induce anti-cancer effects in cancers in digestive organs. J Gastroenterol 2010;45:1097-1102. 